\title{
«L'évaluationite»: symptôme de la désorientation de notre société?
}

\author{
René Raggenbass, membre du Comité central de la FMH
}

Le présent texte ne vise pas l'élimination de l'évaluation en tant que telle mais tente de porter un regard critique sur son renversement actuel; un renversement qui peut, par définition, en faire un instrument de perversion [1].

\section{Evaluation ou I'ère du soupçon contractualisé?}

Aujourd'hui, pour faire face à la phobie des coûts, l'injonction est: évaluez! Evaluer les effets de tout ce que vous faites, vos gestes, vos techniques, vos pensées. Dans le contexte économique et politique actuel, le but recherché semble être l'obtention d'une soi-disant garantie sur la qualité de l'agir. Une garantie justifiée et formalisée au moyen du nouveau triptyque de la «religion contemporaine»: l'efficacité, l'économicité et l'efficience [2]. Cette évaluation est ensuite couronnée par une certification. Une certification qui devrait nous/vous assurer que le partenaire à qui vous avez affaire, celui avec lequel vous voulez contracter, a bel et bien les compétences qu'il prétend avoir! Fini le soupçon! Le contrat ainsi établi - mais évidemment à renouveler - scelle ce délire qui vise la certitude mais qui n'est peutêtre que le symptôme du besoin de légitimation du fragile capitalisme contemporain.

Le corollaire pratique de ce mouvement dans notre clinique est que si vous n'avez pas été évalué et estampillé certifié conforme à la «bonne» pratique vous allez devoir vivre avec le doute et la menace de «malpractice»; même face à un de vos patients qui évolue favorablement [3].

\section{Une évaluation qui ne se fonde que sur les effets (symptômes) est une impasse}

De quel lieu émane la demande d'évaluation, de quelle pratique? Si l'on observe que l'évaluation s'appuie sur un soupçon, une plainte, une critique ou un déficit, personne ne vérifie ni ne s'interroge pour savoir si ces plaintes sont justifiées ou fondées dans une pratique clinique du cas par cas. Aujourd'hui, la «religion» est de répondre à tous les «(m?)effets» de la collectivité et de l'in- dividu par l'évaluation. Dans le domaine de la santé la pression est très clairement mise sur les prestataires avec l'idée sous-jacente que si cela ne fonctionne pas c'est parce qu'ils ne sont pas dans un fonctionnement optimal. Dès lors il n'y a qu'à mesurer, évaluer. On crée et nourrit ainsi le fantasme que «le simple fait d'évaluer constitue l'alpha et l'oméga de la solution. Il suffit alors qu'il y ait eu évaluation pour qu'il y ait déjà solution» [4].

La logique du système est causale et repose sur la dyade problème-solution. Elle se veut pragmatique, objective et vise la suppression de l'effet - symptôme - incommodant. Or, il est facile de démontrer que ce modèle, qui est issu directement de l'industrie, n'est pas adapté lorsqu'il s'agit de questionner les «causes du sujet» [5] et/ou de ses effets. Les mesures faites sur les objets inertes (industriels) sont bien plus faciles que celles faites au contact de la réalité dynamique et vivante d'une situation clinique incarnée à la fois par un sujet malade et par un thérapeute. L'évolution actuelle semble donc plus s'orienter de la mort que du vivant.

\section{Pour une évaluation qui se fonde aussi sur ce qui cause le sujet des effets (symptômes)}

L'observation de ce qui a actuellement cours nous montre que nous sommes en train de renverser l'examen légitime d'une pratique faite dans sa dimension interpersonnelle et dans la logique du cas par cas, par une idéologie de l'évaluation qui se présente comme la solution à un sentiment de «déficit global»; ceci sans même interroger le concept de déficit qui est souvent la seule ou la dernière plus value que certains membres de la collectivité peuvent encore «se payer» [6]. Par conséquent, il est peut-être judicieux que l'on s'intéresse de manière plus ciblée et plus approfondie à la logique, au fond, de ce processus sans quoi nous risquons de produire de plus en plus de symptômes. Il s'agit de développer des instruments spécifiques au domaine de la santé, c'est-à-dire un instrument qui n'écarte pas la subjectivité mais qui l'inclut comme paramètre. Seule une évaluation qui intègre les spécificités 
du cas par cas peut nous orienter sur ce qui cause le sujet et ses effets, et ouvrir la réflexion vers de nouvelles perspectives économiques, politiques et sociales.

\section{S'orienter au-delà de l'effet qui fait symptôme}

L'orientation de l'évaluation actuelle cherche à faire disparaître le seul objet qui la dérange, lui fait obstacle et la met en échec: le sujet. Dans le domaine de la santé, la singularité ne se laissera jamais totalement enfermer dans la généralisation d'une évaluation, de guidelines ou d'autres cadres. Dans cette évaluation industrielle, ce qui fera toujours excès, c'est la dimension contingente du vivant. Or, lorsque la collectivité ne s'oriente pas de ce qui cause le sujet, elle se disperse en s'orientant des multiples discours (de pseudo savoirs) qui risquent de ne devenir que des discours de légitimation incarnés par des anxieux supposés savoirs qui ne font que problématiser à l'infini les effets (symptômes) au détriment du lien social.

\section{«Evaluationitis»: ein Symptom der Orientierungslosigkeit unserer Gesellschaft?}

René Raggenbass, Mitglied des Zentralvorstandes der FMH

1 Perversion bedeutet ja so viel wie Umkehrung.

2 Als hätte es vorher ein bewusstes Zeitalter der Wirkungslosigkeit, Unwirtschaftlichkeit und Ineffizienz gegeben?

3 Wir wissen jedoch aus dem Klinikalltag, dass auch trotz richtigen Handelns manchmal die Entwicklung bei einem Patienten ungünstig verläuft. In diesem Fall kann der Therapeut aber ruhigen Gewissens schlafen! Die Tyrannei der Evidence-based Medicine, die sich auf eine Hierarchisierung der Beweise stützt und seit Beginn der 90er Jahre vorherrschend ist, stösst allerdings langsam ebenfalls an ihre Grenzen.
Dieser Text will nicht die Abschaffung der Evaluierung an sich anvisieren, sondern versucht, einen kritischen Blick auf ihre Verkehrung ins Gegenteil zu werfen; Verkehrung kann durchaus zu einem Instrument der Perversion [1] werden.

\section{Evaluierung oder vertraglich institutionalisiertes Misstrauen?}

Angesichts der Kostenphobie werden heutzutage überall Evaluierungen befohlen. Evaluiert werden soll die Wirkung von Handlungen, Techniken und Gedanken. Im aktuellen wirtschaftlichen und politischen Umfeld scheint der Zweck darin $\mathrm{zu}$ bestehen, gewissermassen eine Garantie für die Qualität des eigenen Handelns zu erhalten.

Diese Garantie soll mit Hilfe des neuen Triptychons der «zeitgenössischen Religion», nämlich Wirksamkeit, Wirtschaftlichkeit und Effizienz, gerechtfertigt und formalisiert werden [2]. Die Evaluierung wird anschliessend noch durch eine Zertifizierung gekrönt. Diese soll uns und Ihnen bescheinigen, dass der Partner, mit dem Sie es zu tun haben und mit dem Sie einen Vertrag abschliessen wollen, über die Kompetenzen verfügt, die er zu haben behauptet. Damit ist dann jegliches Misstrauen beseitigt! Der so geschlossene, aber selbstverständlich später wieder zu erneuernde Vertrag besiegelt diesen Sicher- heitswahn, der jedoch vielleicht nur ein Symptom des Legitimierungsbedürfnisses des anfälligen Kapitalismus von heute darstellt.

Die praktische Folge dieser Entwicklung in unserem Klinikalltag besteht darin, dass Sie ohne eine Evaluierung und den Zertifizierungsstempel mit der Konformitätsbescheinigung bezüglich des «guten» Praktizierens mit dem Zweifel und der Gefahr der «malpractice» leben müssen selbst bei einem Patienten, bei dem die Entwicklung günstig verläuft [3].

\section{Eine ausschliesslich auf die Auswirkung (Symptome) ausgerichtete Evaluierung führt in die Sackgasse}

Woher und aus welcher Praxis stammt der Ruf nach Evaluierung? Wenn die Evaluierung sich auf einen Verdacht, eine Klage, eine Kritik oder eine Unzulänglichkeit stützt, prüft niemand nach und niemand fragt, ob diese Beschwerde gerechtfertigt oder in einer fallweisen Betrachtung der klinischen Praxis begründet ist. Das heutige Credo lautet, auf alle (negativen) Reaktionen der Allgemeinheit oder des einzelnen mit einer Evaluierung zu antworten. Im Gesundheitswesen wird ganz klar Druck auf die Leistungserbringer ausgeübt mit dem Unterton: Wenn etwas nicht funktioniert, liegt der Grund 
4 Miller J-A, Milner J-C. Voulez-vous être évalué? Paris: Figures/Grasset; 2004.

5 Das, was das Subjekt sagt, ist nicht deckungsgleich mit dem, was es denkt. Die das Handeln des Subjekts bestimmenden Ursachen sind der Ursprung dessen, was wir sind. Diese grösstenteils unbewussten Elemente strukturieren unsere Persönlichkeit. Man darf nicht Ursache und Wirkung im Handeln eines Subjekts verwechseln (d.h., was es sagt, tut und/oder was für Symptome es aufweist). Eine Messung der Wirkung sagt folglich nichts über die Ursache aus.

6 Diese Feststellung beschränkt sich nicht auf den Klinikalltag, sondern wird auch durch eine 2002 in der Zeitschrift «Nature» veröffentlichte Studie belegt. Darin wird aufgezeigt, dass «eine Person es akzeptieren kann, zum Wohl der Gruppe schwächer zu werden». Siehe Arbeit von S. Gächter, Professor für Wirtschaftspolitik an der Universität St. Gallen und Träger des Latsis-Preises. darin, dass sie nicht optimal funktionieren. Dann braucht man ja nur zu messen und zu evaluieren. So schafft und pflegt man die irrige Vorstellung, dass «allein die Tatsache, dass man evaluiert, bereits das $A$ und $O$ der Lösung darstellt. Es reicht dann, eine Evaluierung durchgeführt zu haben, um bereits die Lösung präsentieren zu können» [4].

Das System verfügt über eine Kausallogik und basiert auf dem Begriffspaar Problem und Lösung. Es will pragmatisch und objektiv sein und die lästige Auswirkung - das Symptom - beseitigen. Man kann leicht beweisen, dass dieses direkt aus der Industrie stammende Modell nicht geeignet ist, um das zu hinterfragen, was das Handeln des Subjekts bestimmt (Ursache) und welche Wirkung dieses Handeln hat [5]. Messungen an leblosen (industriellen) Gegenständen sind viel einfacher als solche im Kontakt mit der dynamischen und lebendigen Wirklichkeit einer klinischen Situation, in die ein Patient und ein Therapeut involviert sind. Die aktuelle Entwicklung scheint sich folglich eher am Toten als am Lebendigen zu orientieren.

\section{Für eine Evaluierung, die sich auch mit dem befasst, was das Handeln des Subjekts und seine Wirkung (Symptome) bestimmt}

Wenn wir beobachten, was zurzeit Gültigkeit hat, dann sehen wir, dass wir dabei sind, die legitime Überprüfung einer Praxis in ihrer zwischenmenschlichen Dimension und der Logik der fallweisen Betrachtung durch eine Evaluierungsideologie zu ersetzen, die sich als Lösung für das Gefühl eines «allgemeinen Defizits» präsentiert. Dabei hinterfragen wir diesen Begriff des Defizits nicht, der oft den einzigen oder letz- ten Mehrwert darstellt, den sich gewisse Personen noch «leisten» können [6]. Folglich wäre es vielleicht angebracht, sich zielgerichteter und eingehender mit der Logik und dem Inhalt dieses Prozesses zu befassen, denn sonst laufen wir Gefahr, immer mehr Symptome zu produzieren. Es geht darum, spezifisch auf das Gesundheitswesen zugeschnittene Instrumente zu entwikkeln, welche die Subjektivität nicht ausschliessen, sondern als Parameter berücksichtigen. Nur eine Evaluierung, welche die Besonderheiten der fallweisen Betrachtung einschliesst, kann uns einen Hinweis auf Ursache und Wirkung geben und Überlegungen hinsichtlich neuer wirtschaftlicher, politischer und gesellschaftlicher Perspektiven in Gang setzen.

\section{Ein Ansatz, der über die zum Symptom gewordene Auswirkung hinausgeht}

Die aktuelle Ausrichtung der Evaluierung versucht, den einzigen störenden Gegenstand zu beseitigen, der sie scheitern lässt: das Subjekt. Im Gesundheitswesen kann die Einzigartigkeit niemals völlig ins verallgemeinernde Korsett einer Evaluierung, von Richtlinien oder anderen Rahmenbedingungen gezwängt werden. Bei dieser industriellen Evaluierung wird die kontingente Dimension des Lebendigen immer das sein, was den Rahmen sprengt. Wenn die Allgemeinheit sich nicht daran orientiert, was das Handeln des Subjekts bestimmt, verzettelt sie sich, indem sie sich von verschiedenen Diskursen (Pseudowissen) leiten lässt, die möglicherweise nur der Legitimierung des Diskurses über angstgeleitetes Scheinwissen dienen, das jedoch - zulasten der sozialen Beziehung - lediglich die Auswirkung (Symptome) bis ins Unendliche problematisiert. 